عبدالمجيد جرجانى '، حسن اكبرى '، سيد عطاله حسينى 'و اميد عبدى

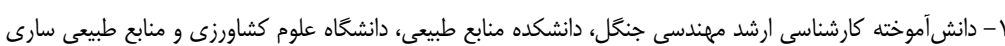

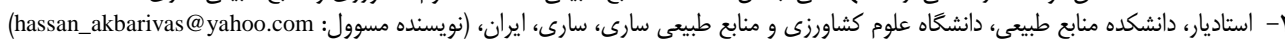

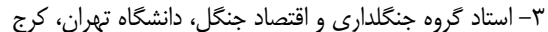

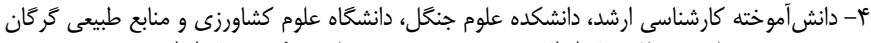
تاريخ بذيرش:

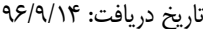

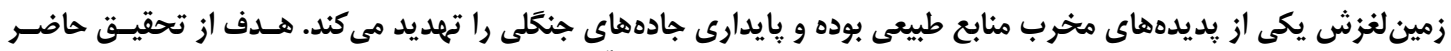

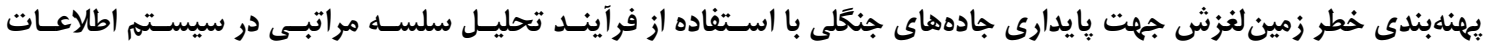

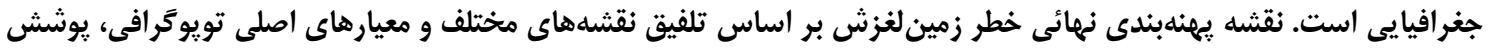

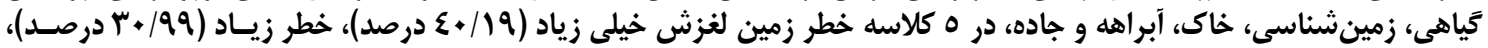

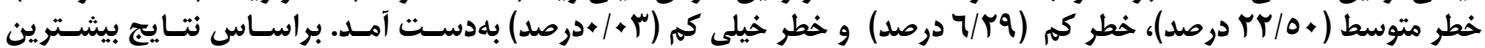

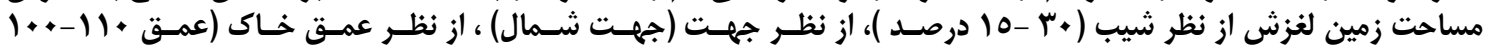

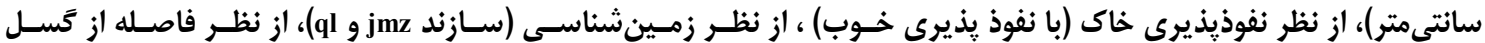

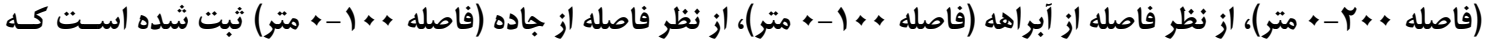

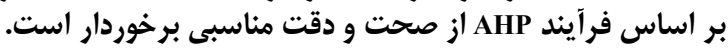

وازههاى كليدى: تحليل سلسله مراتبى، سيستم اطلاعات جغرافيايى، معيار اصلى، جاده جنكلى، لغزش، نقشه يهنهبندى

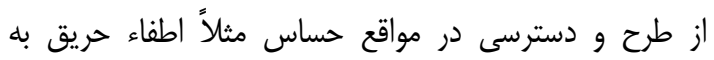

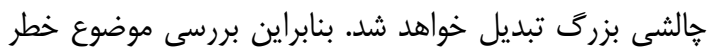

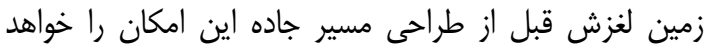

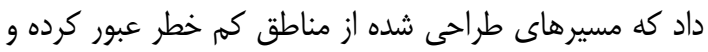

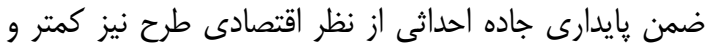

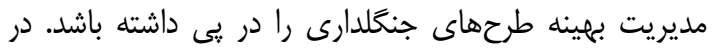

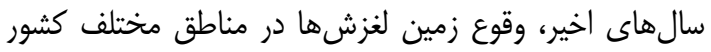

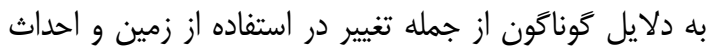

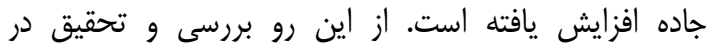

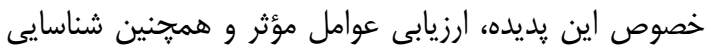

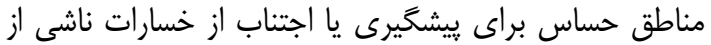

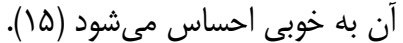

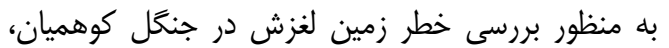

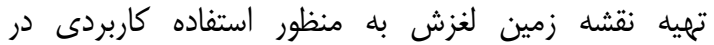

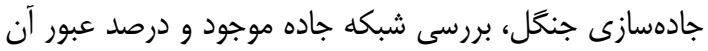

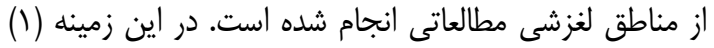

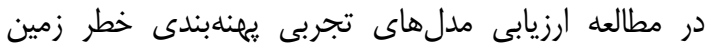

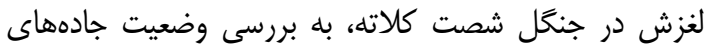
طراحى شده جنغل شصت كلاته در در محيط

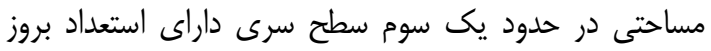

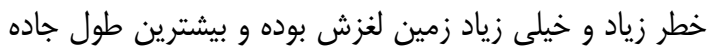

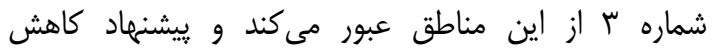

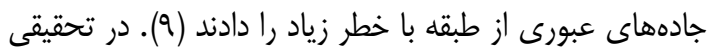

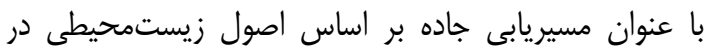

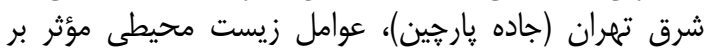

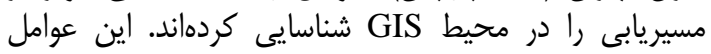
عبارتند از زمينشناسى، فرسايشيذيرى خاك، شيب، كاربرى

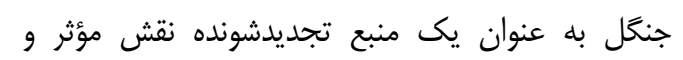

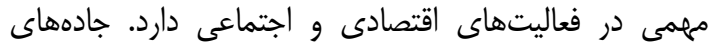

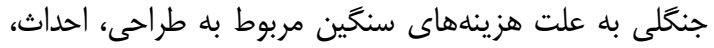

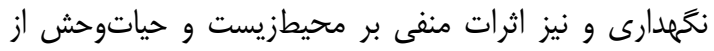

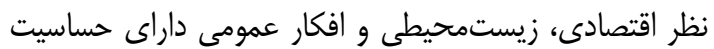

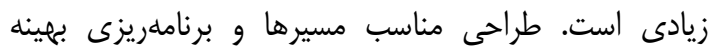

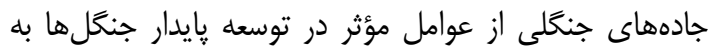

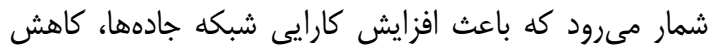

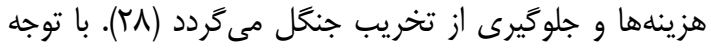

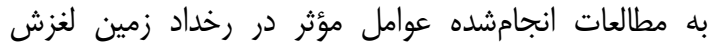

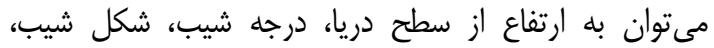

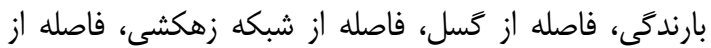

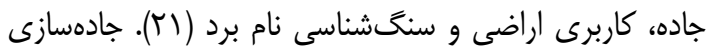

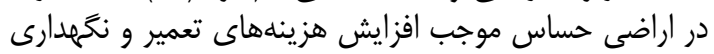

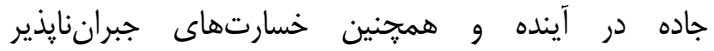

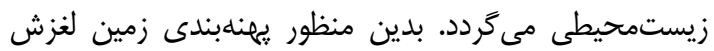

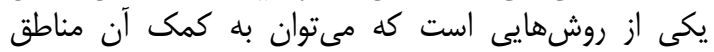

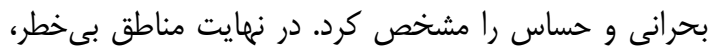

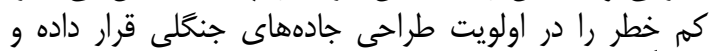

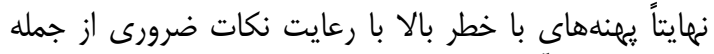

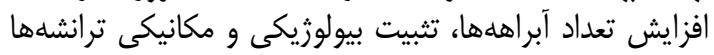

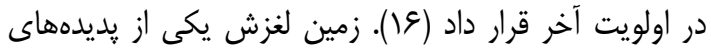

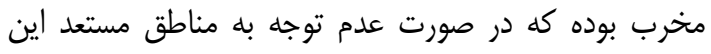

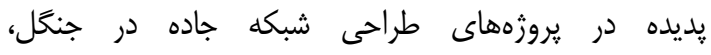

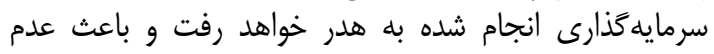
سرويسدهى مناسب به امور مديريتى طرح بخصوص خدار حفاظت 
شيب، جهت شيب، يوشش گياهى، زهكشى و فاصله از جاده

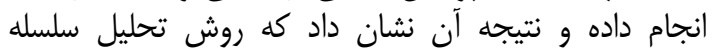

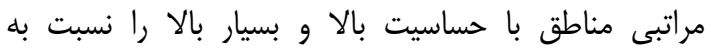

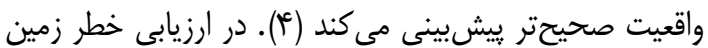

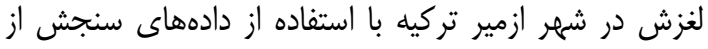

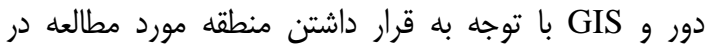

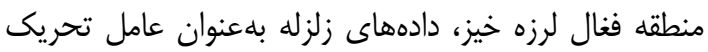

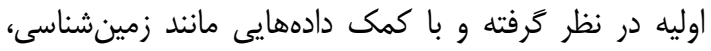

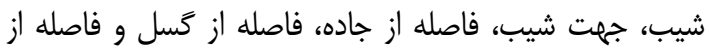

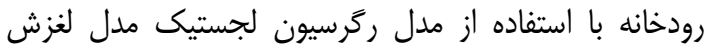

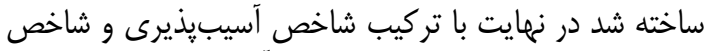

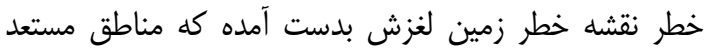

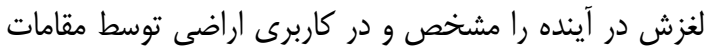

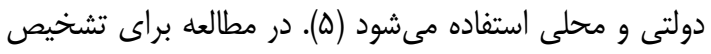

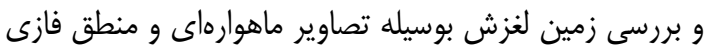
در غرب درياى سياه تركيه دريافتند كه نقشه تولئ توليد شده توسط

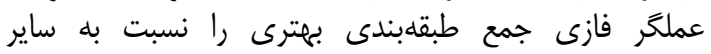

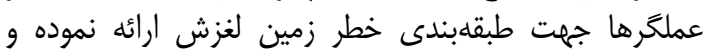

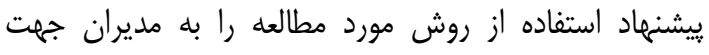

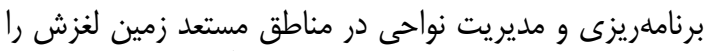

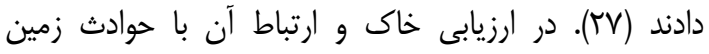

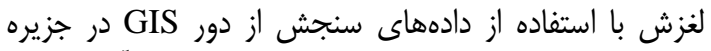

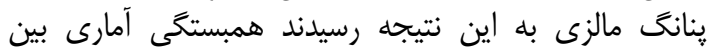

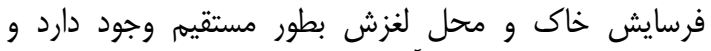

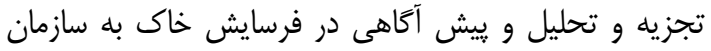

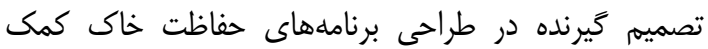

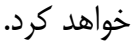

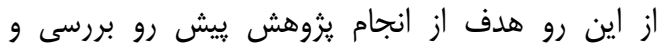

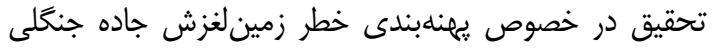

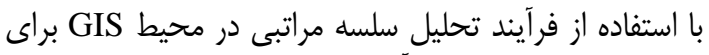
طرح جنكلدارى كوهميان آزادشهر است.

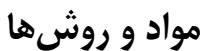

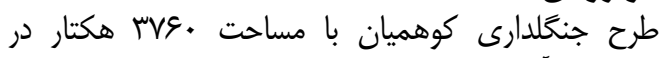

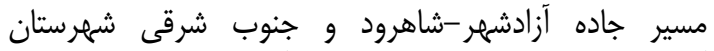

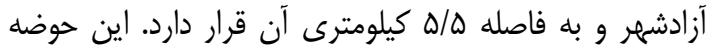

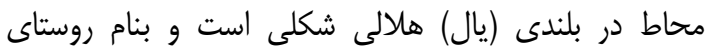

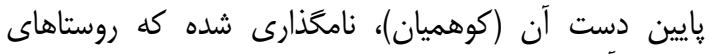

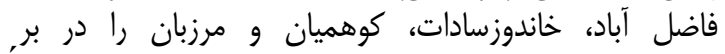

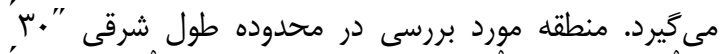

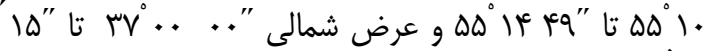

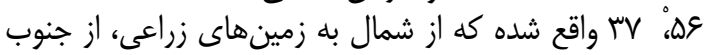

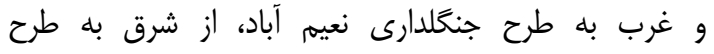
جنحَلدارى وطن متصل مى باشد (شكل (1).
اراضى، جريانهاى آبى، كَل و ارتفاع، نقشههاى كاربرى

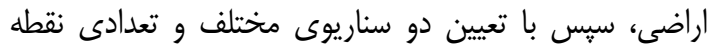

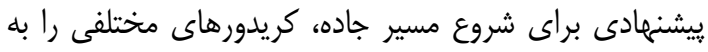

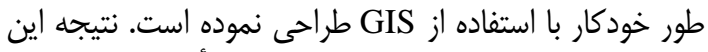

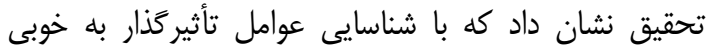

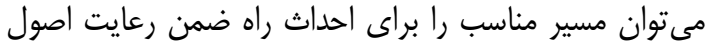

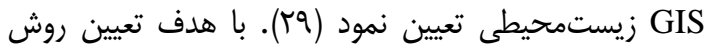

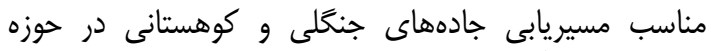

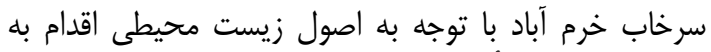

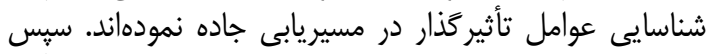

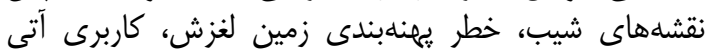

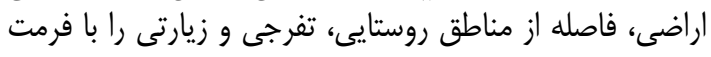

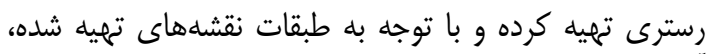

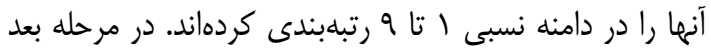

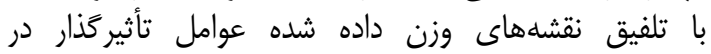

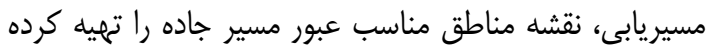

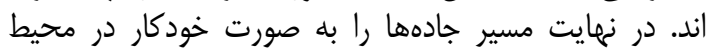
GIS

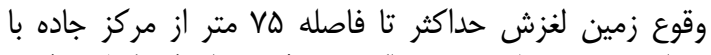

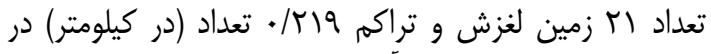

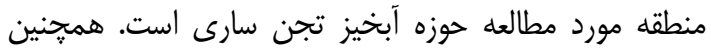

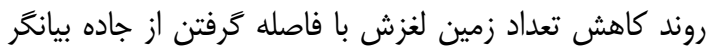

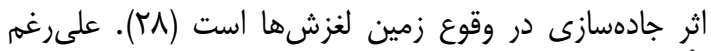

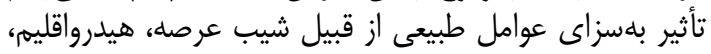

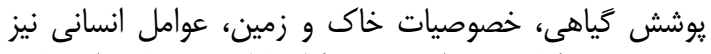

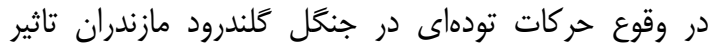

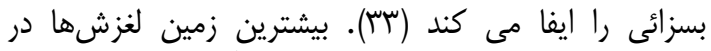

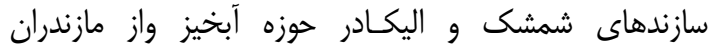

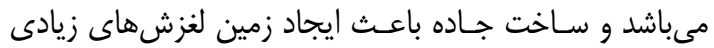

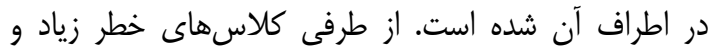

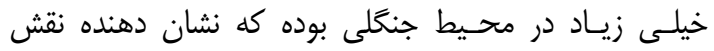

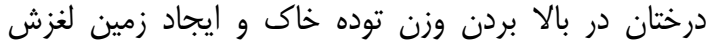

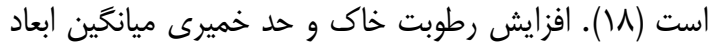

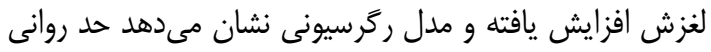

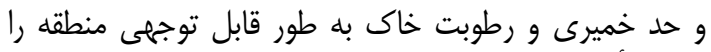

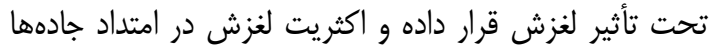

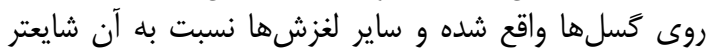

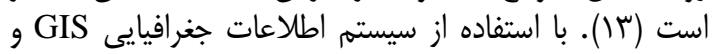

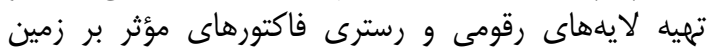

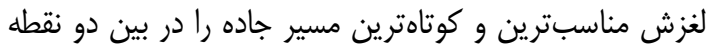

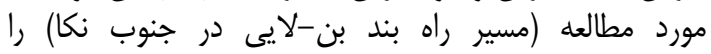

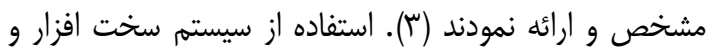

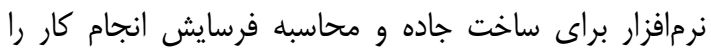

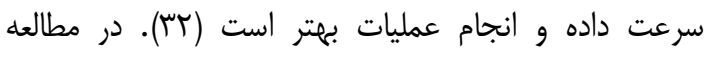

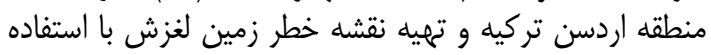

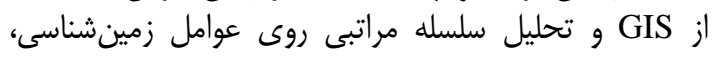



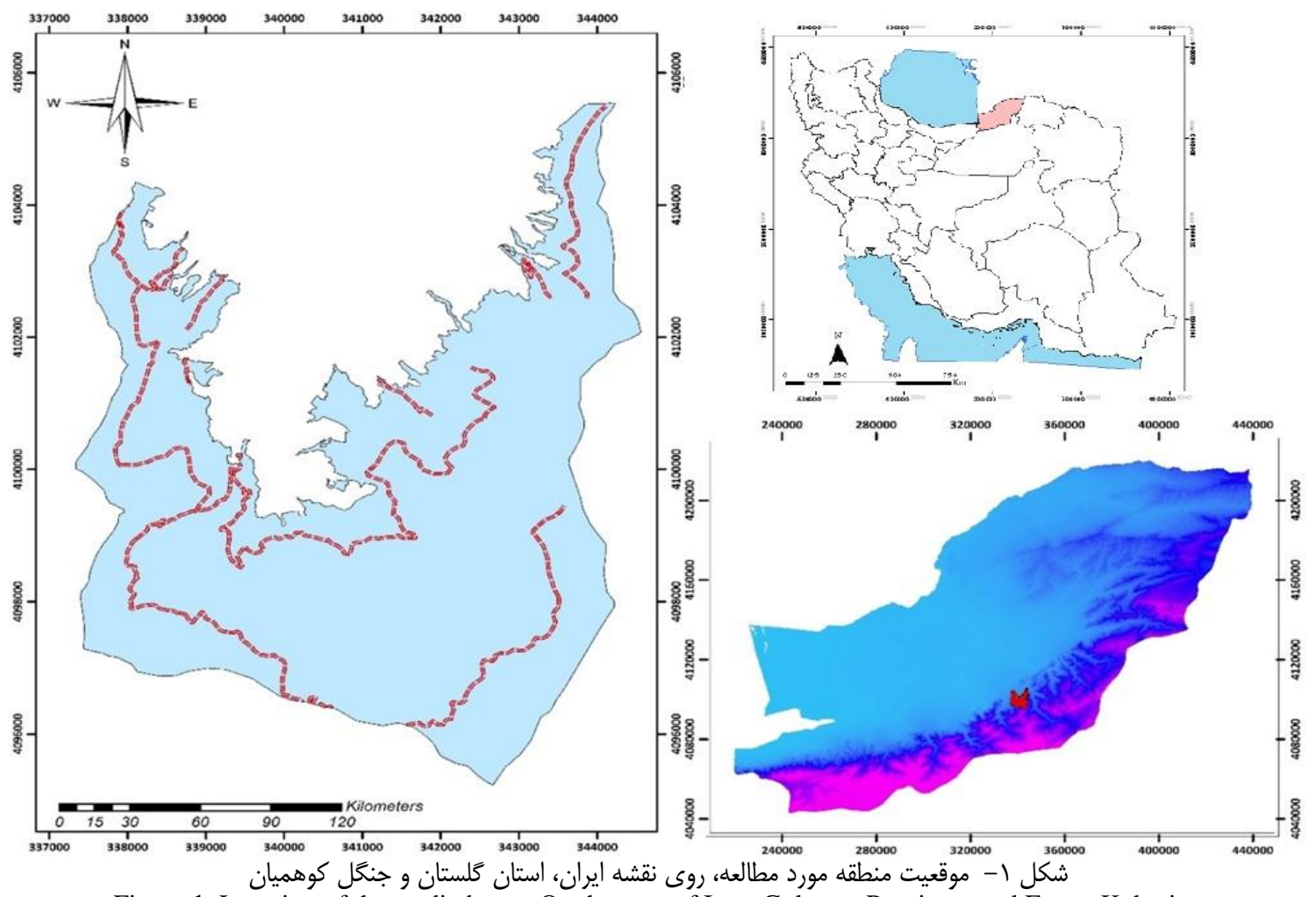

Figure 1. Location of the studied area ,On the map of Iran, Golestan Province; and Forest Kohmian

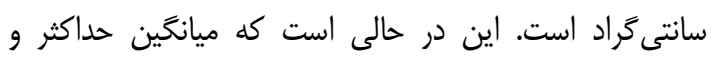

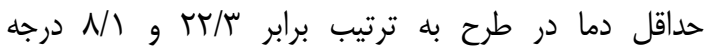

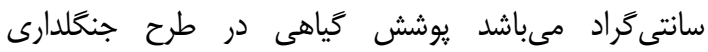
كوهميان شامل كَونهاى

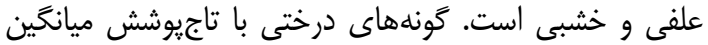

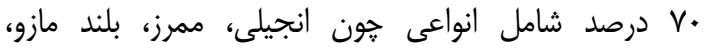

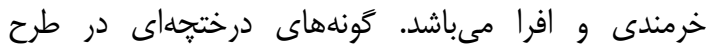

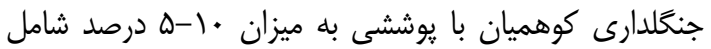

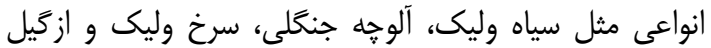

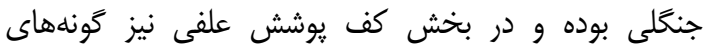

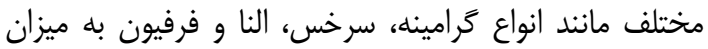
•r الى •ه درصد (اوايل سال بيشتر از ساير فصول است)

$$
\text { حضور دارند (•r) (r). }
$$

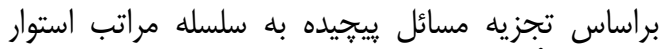

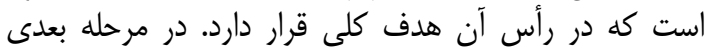

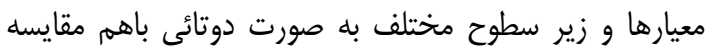

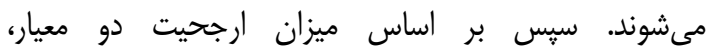

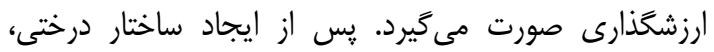

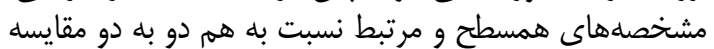

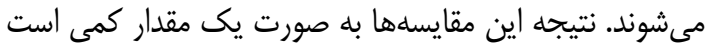

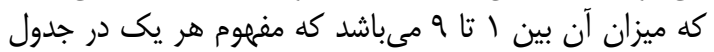

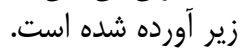

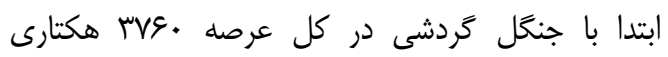

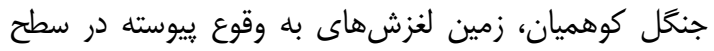

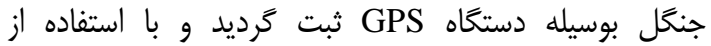

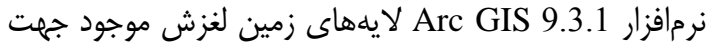

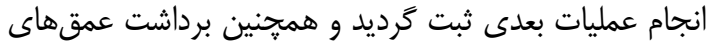

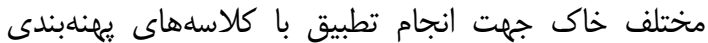

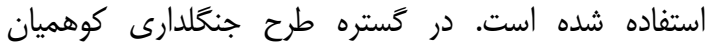

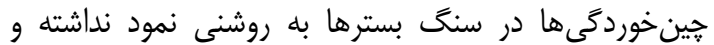

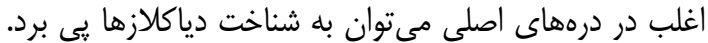

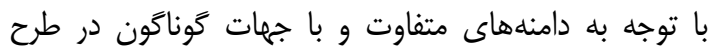

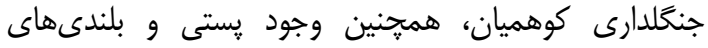

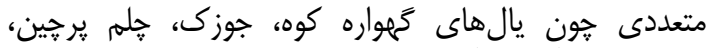

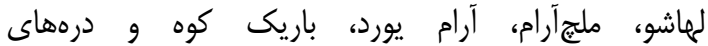

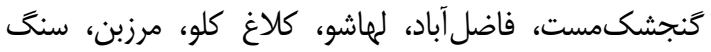

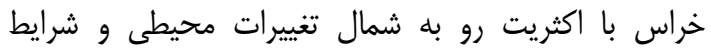

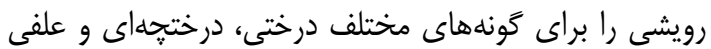

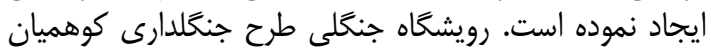

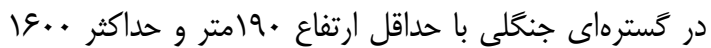

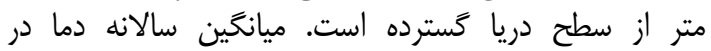

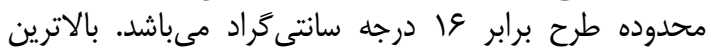

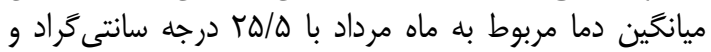

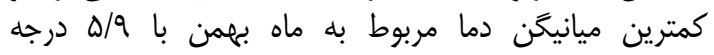


Table 1. Preferences questionnaire

جدول 1- ترجيحات يرسشنامه

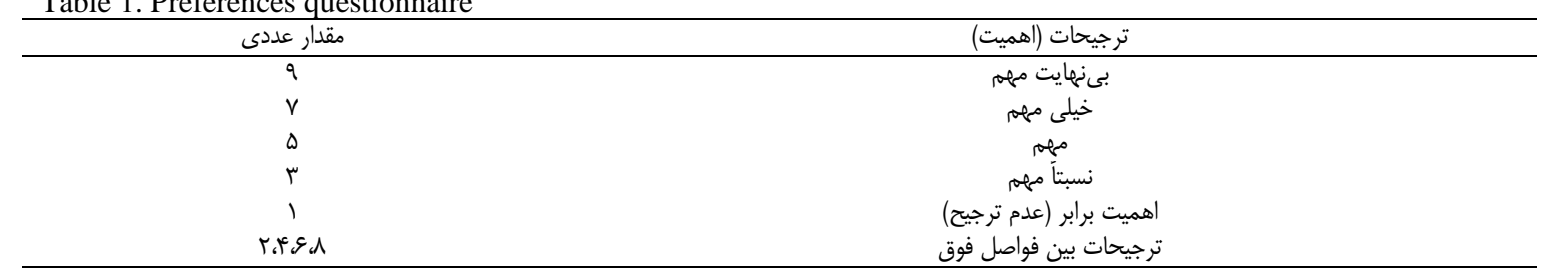

يوشش گَياهى، تويوگرافى، مسير جاده و آبراهه تهييه شد. بر آبر

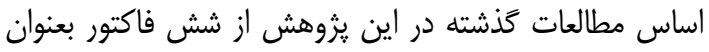

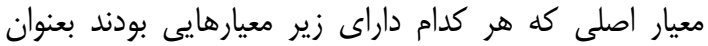

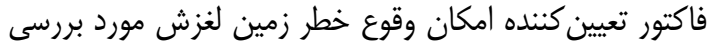

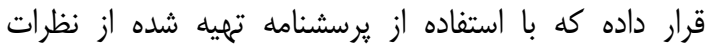

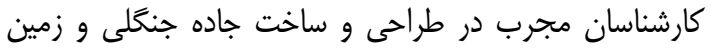

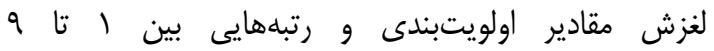

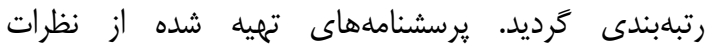
كارشناسان جهت مقايسه دوبه دو عوامل براى برى برسئ و و تعيين

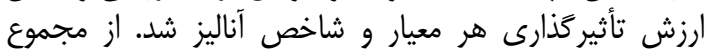

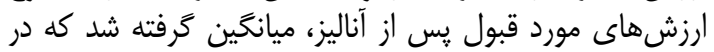

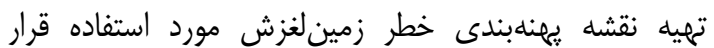

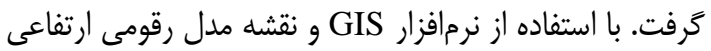

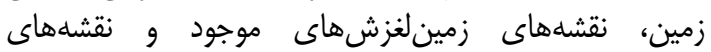

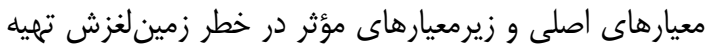

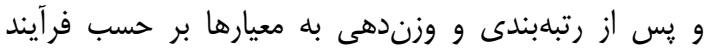

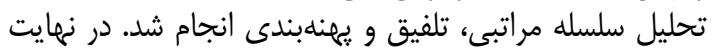

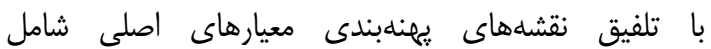

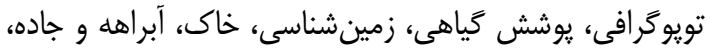

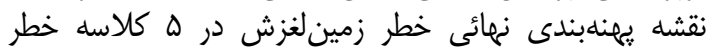
زمين لغزش بهدست آمد.
يرسشنامههاى تهييه شده از نظرات كارشناسان مجرب در در

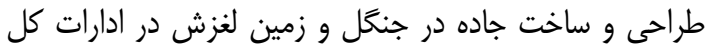

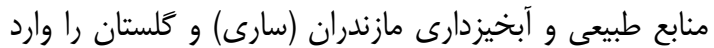

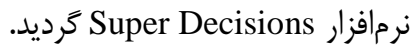

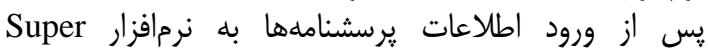
Decision

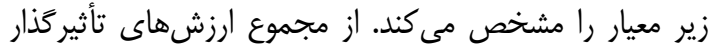

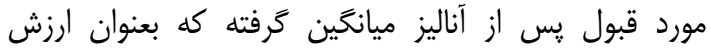

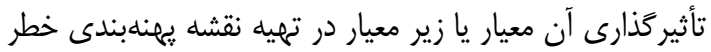
زمين لغزش استفاده خواهد شئ أندار

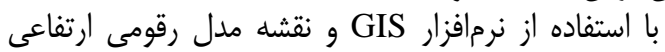

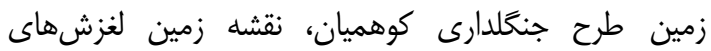

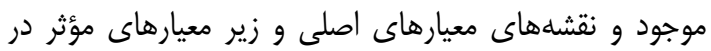

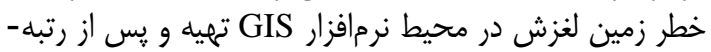

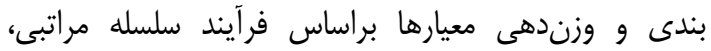

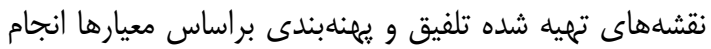

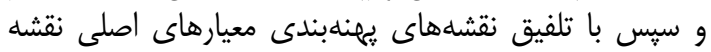

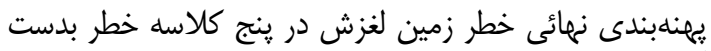

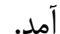

بلهنظور انجام مراحل تحقيق : برسشنامهاى توسط تيم

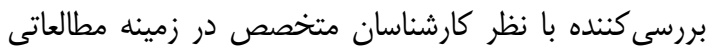

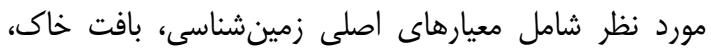

جدول r- وزن عوامل تأثير كذار بر خطر زمين لغزش در طرح جنحلدارى كوهميان Table 2. Weight of factors influencing the risk of landslide in the Kohimish forestry plan

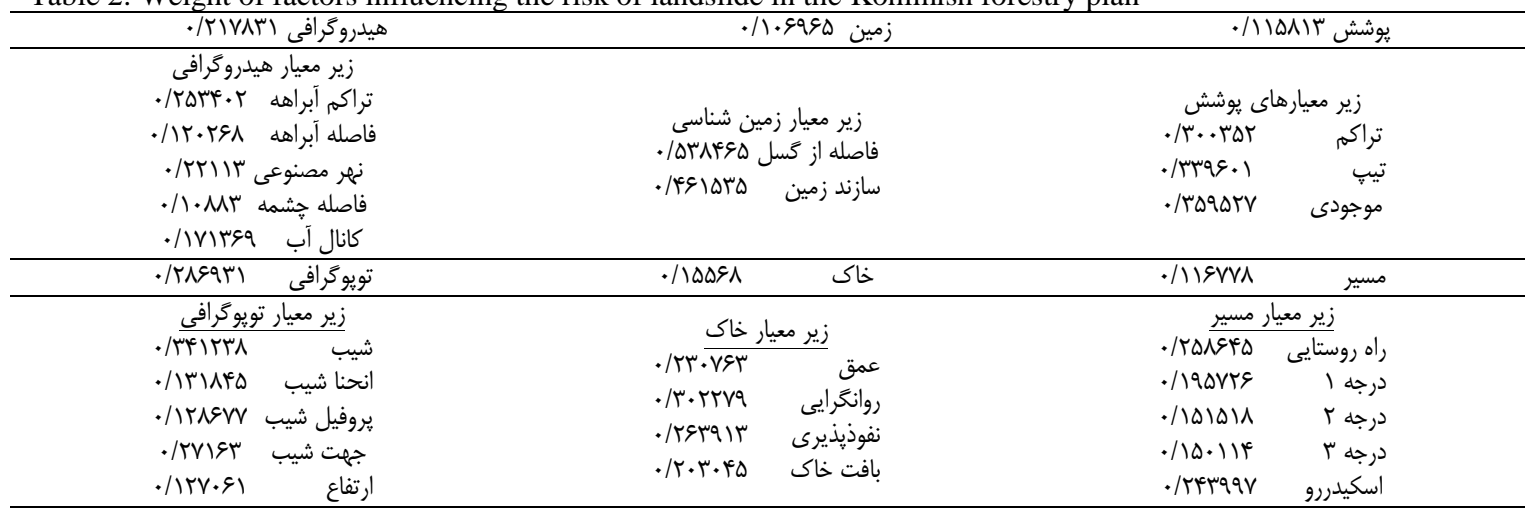

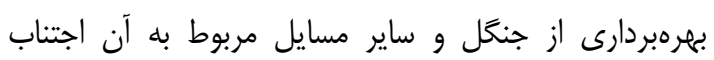

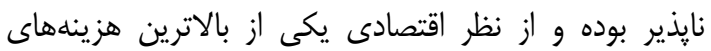

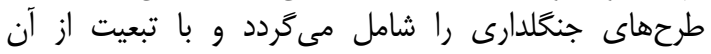

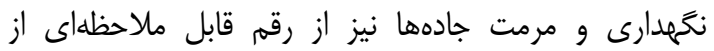

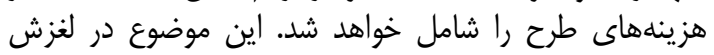

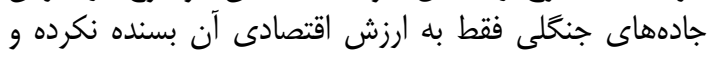

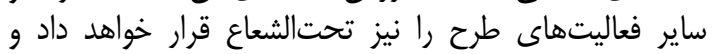

در اين يثوهش به بررسى عوامل مؤثر در خطر زمين

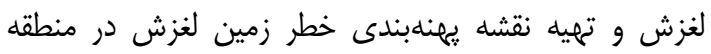

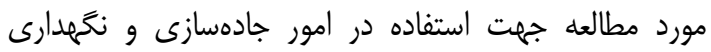

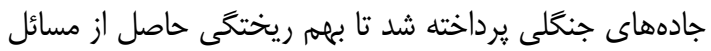

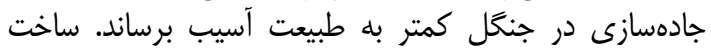

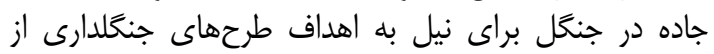

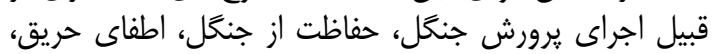


شيبهاى كمتر از ها درصد به دليل كاهش نيروى ثقل و

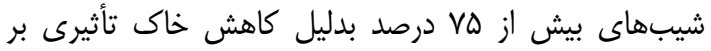

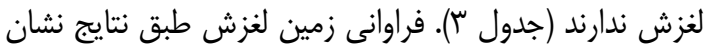

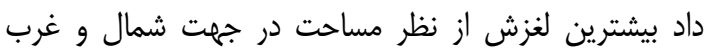

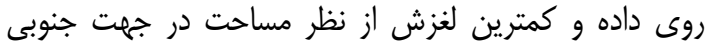

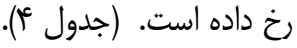

بيشتر نيز با حركت خاك و زمين باعث فرسايش و از بين رفتن خاك را نيز فراهم مىنمايد.

نتايج و بحث

نتايج نشان داد بيشترين لغزش از نظ نظر مساحت در طبقه

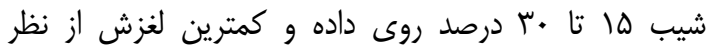

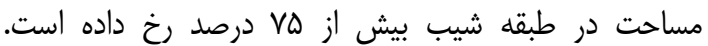

Table 3. Risk of landslide hazard in slope categories

جدول س- رتبهبندى خطر زمين لغزش در طبقات شيب

\begin{tabular}{|c|c|c|c|c|c|}
\hline رتبه خطر زمين لغزش هر & نسبت لغزش به نسبت كل & تعداد يِيكسل لغزش & ي ي تعسل هاد & مساحت هكتار & طبقات شيب \\
\hline$\Delta$ & ./rrrqq.r+人 & $M \mu$ & $\Lambda I V$ & MYN/FA & $\cdot-10$ \\
\hline f & . & FVA & rAItr & $\| r \Delta / r \Lambda$ & |Q-r. \\
\hline r & . . .ququाf & TEV & TAFTA & $11 \% \mathrm{~V}$ & $f \Delta-r$. \\
\hline r & 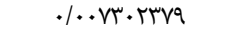 & ir. & سזקופו & SDV/Tr & $f \Delta-q$. \\
\hline 1 & $\cdot$ & - & SVG. & $r v \cdot / r$ & $\varepsilon \cdot-V \Delta$ \\
\hline 1 & - & . & $r \cdot \Delta V$ & $\Lambda T / T \Lambda$ & $>V \Delta$ \\
\hline
\end{tabular}

Table 4. Landslide risk rating table in slope steps

\begin{tabular}{|c|c|c|c|c|c|}
\hline رتبه خطر زمين لنزش هر طبقه & نسبت لغزش به نسبت كل & تعداد ييكسل لغزش & تعداد يِيكسلها & مساحت هكتار & طبقات جهت شيب \\
\hline r & ./. MTEITESV & Far & SAla & $\mid f \Delta s / \varphi$ & شمال \\
\hline r & $\cdot /\left.\cdot \Delta \Delta \cdot\right|^{c}$ & $\wedge$ & IASTA & gra & 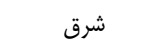 \\
\hline r & . $/ . M T H^{2}$ & v & MITD & ITa & جنوب اب \\
\hline$\Delta$ & . IFTIT.DFD & $\Delta \cdot r$ & rorq & E & غرب \\
\hline
\end{tabular}

كه به تبع آن لغزش نيز بيشتر مشاهده گرديده است. از نظر

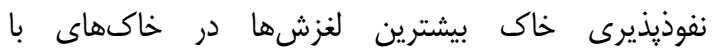

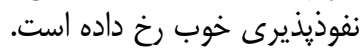

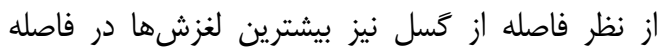

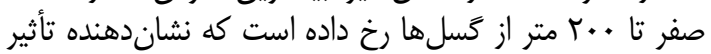

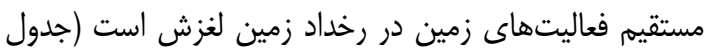

از نظر عمق خاك و لغزشهاى صورت يذيرفته بيشترين

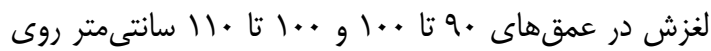

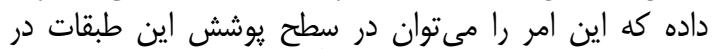

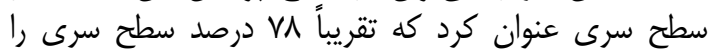

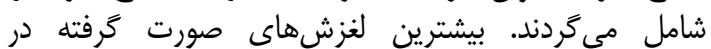

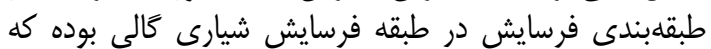

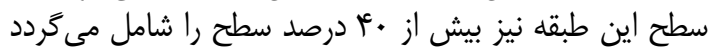

جدول ه- رتبهبندى خطر زمين لغزش در طبقات عمق خاى (كتابحه طرح جنكلدارى) (Anonymous, 2011) Table 5. Landslide risk rating in depths of soil

\begin{tabular}{|c|c|c|c|c|c|}
\hline رتبه خطر زمين لغزش هر طبقه & نسبت لغزش به نسبت كل & تعداد ييكسل لغزش & تعداد يِيكسل ها & مساحت هكتار & طبقات عمق خاك CM \\
\hline 1 & . +..VVDAYAV & $r$ & rqVर & $1 Q N / M$ & $>11$ \\
\hline r & $+1+V \Delta 9 \Delta \cdot 9)^{c}$ & 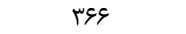 & Fᄉ।19 & $19 \Delta V / \Delta S$ & $11 \cdot-1 \ldots$ \\
\hline$\Delta$ & . - trtaitieie & DIf & rTDAT & $q \cdot r / \& \wedge$ & $1 \cdots-9$ \\
\hline f & . & ITA & DMAT & rTa/ra & $V \Delta-q$. \\
\hline r & . . . rVSG9V & rv & 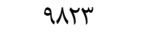 & rar/ar & كمتر از VD \\
\hline
\end{tabular}

جدول צ- رتبهبندى خطر زمين لغزش در طبقات نفوذيذيرى خاى (كتابِه طرح جنگلدارى) (Anonymous, 2011) Table 6. Landslide risk rating in soil permeability classes

\begin{tabular}{|c|c|c|c|c|c|}
\hline رتبه خطر زمين لغزش هر طبقه & نسبت لغزش به نسبت كل & تعداد ييكسل لغزش & تعداد يِيكسلها & مساحت هكتار & طبقات نفوذيذيرى خاى \\
\hline r & .1. gVrefeq & ITV & trr & $V I r / \& \Lambda$ & ضعيف تا متوسط \\
\hline$\Delta$ & $\cdot 1 \cdot 10 \cdot+r 109$ & Fiv & rrvas & $1111 / \lambda \mathrm{F}$ & خوب \\
\hline
\end{tabular}


جدول V- - رتبهبندى خطر زمين لغزش در طبقات فاصله از گسل (كتابجه طرح جنگلدارى) (Anonymous, 2011) Table 7. Landslide hazard rating in the distance classes of faults

\begin{tabular}{|c|c|c|c|c|c|}
\hline رتبه خطر زمين لغزش هر طبقه & نسبت لغزش به نسبت كل & تعداد يِيكسل لغزش & تعداد يِيكسلها & مساحت هكتار & طبقات فاصله از گسل متر \\
\hline$\Delta$ & $.1 \cdot 19 V V \Delta \wedge V \pi$ & rq. & I9VTI & VMN/AF & $r \cdots-\cdot$ \\
\hline r & . +. VIVarVie & (r & IOTYT & $V E N / M$ & $r \cdots-r \cdots$ \\
\hline f & . - IDVDFFQ9 & rII & س ( & QTL/VT & $\| r \cdot-\lambda \cdots$ \\
\hline r & $\cdot / \cdot$ HEAV.NT & th & 9 & TAT/RE & $\mid \varepsilon \cdots-14 \cdot$. \\
\hline
\end{tabular}

تاج يوشش رخ داده است كه اين موضوع نسبت مستقيم با

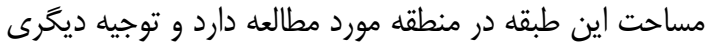

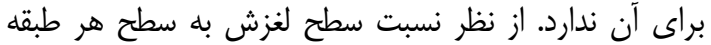

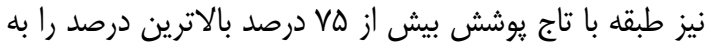

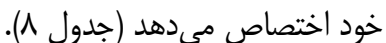

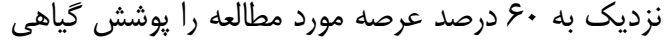

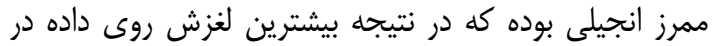

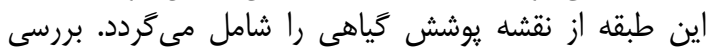

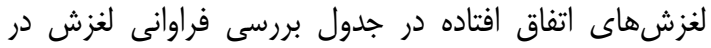

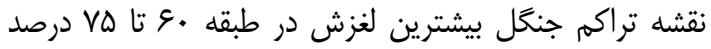

Table 8. Landslide hazard zonation results in Vegetation Criteria

جدول ^-نتايج يهنهبندى خطر زمين لغزش در معيار يوشش گياهى

\begin{tabular}{|c|c|c|c|}
\hline طول جاده (متر) & درصد عبور جاده & مساحت هكتار & كلاسه خطر \\
\hline$\Gamma \Delta \wedge \Delta / Q \Delta V T \Delta \&$ & W/VTEDFEGT & $1 \cdot N / 4$ & خطرخيلى كم \\
\hline$V \Delta \Delta F / \Delta T / 99 V$ & rF/V. FrqVHr & $1 . \mu 1 / \mu r$ & خطر كم \\
\hline SVKT/.rIATI & rI/QADLARIV & $1 . .8 / M$ & خطر متوسط \\
\hline IV.D/QITAYG & $\triangle / \Delta V \wedge \Delta \Delta \Delta Q V I V$ & FIF/AF & خطر زياد \\
\hline $11.1 . / \mathrm{raATV}$ & $r s / . \Delta A T|\Lambda| F$ & $1 . \Delta T / r g$ & خطر خيلى زياد \\
\hline
\end{tabular}

آبشوئى در هنگام سيلابى شدن كناره رودخانها و و برداشت

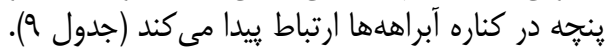

در بررسى جدول فراوانى لغزش در طبقات فاصله از آبراههها

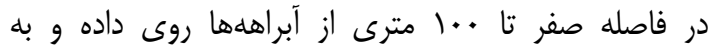

Table 9. Landslide hazard rating table in the distance between the waterways

جدول q- جدول رتبلهندى خطر زمين لغزش در طبقات فاصله از آبراهه

\begin{tabular}{|c|c|c|c|c|c|}
\hline رتبه خطر زمين لغزش هر & نسبت لغزش به نسبت كل & تعداد بيكسل لغزش & تعداد بيكسل ها & مساحت هكتار & طبقات فاصله از آبراهه (متر) \\
\hline$\Delta$ &.$/ .191 \Delta 91 V^{\mu}$ & FVI & THVIV & $94 N / 9 \Lambda$ & $1 \cdots \cdot$ \\
\hline r &.$/ .+a q \Delta \Delta \Delta q 1$ & 90 & 195.9 & VEN/RE & $r \cdot \cdot-1 .$. \\
\hline r & . . & $10 \Delta$ & $109 \Delta \Delta$ & GHN/T & $r \cdots-r \cdots$ \\
\hline f & . $1 \cdot 1 r+4 \cdot r q$ & IMF & $1 \cdots \mathrm{r} \wedge$ & $r \cdot r / \mu r$ & r...r... \\
\hline f & . & va & syei & $r \& q / 9 r$ & $\Delta \cdots-f \cdot$. \\
\hline f & . & IrA & 11899 & eVI/ag & $1 \cdots-\Delta \cdots$ \\
\hline 1 & • & . & rqure & $11 V /{ }^{\prime} \varepsilon$ & $>1 \ldots$ \\
\hline
\end{tabular}

خوردن عرصه با جادهسازى بوده و يا عدم رعايت إداد اصول فنى إنى

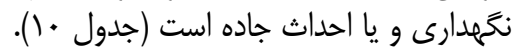

لغزش هاى رخ داده در جدول طبقات فاصله از جاده نيز همانند

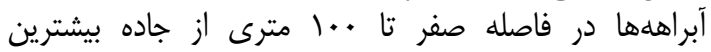

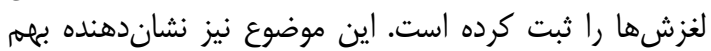

Table 10. Landslide risk rating in the distance between roads and roads

جدول • ا- رتبهبندى خطر زمين لغزش در طبقات فاصله از مسير جاده

\begin{tabular}{|c|c|c|c|c|c|}
\hline رتبه خطر زمين لغزش & نسبت لغزش به نسبت كل & تعداد يِيكسل لغزش & تعداد يِيكسلها & مساحت هكتار & طبقات فاصله از مسير جاده (متر) \\
\hline$\Delta$ & $\cdot / \cdot r V q \cdot 1 \cdot r \Delta$ & FID & IfAVte & $\Delta q 4 / 9 q$ & $1 \cdots-\cdot$ \\
\hline r & $\cdot \cdot \cdot V T H \cdot F \Delta q$ & vq & 1.948 & $\mathrm{frV} / \cdot \mathrm{F}$ & $r \cdots-1 \ldots$ \\
\hline 1 & . +. NVERgF & ب ب & $91 \cdot r$ & rqr/Ir & r..-r.. \\
\hline r & | & $v^{e}$ & $M \cdot 9$ & TQYTKF & ץ...r... \\
\hline 1 & . . . FArgVRr & qq & $\Lambda \cdot \wedge$. & ( & $\Delta \cdots-\uparrow \cdots$ \\
\hline r & . . . qVeragV & rq. & rqsqu & $1191 / 94$ & $1 \cdots-\omega \cdots$ \\
\hline f & 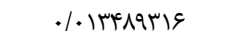 & سו & ᄉrVV & 1 & $>1 \ldots$ \\
\hline
\end{tabular}


نقشه كلاسه بندى خطر يوشش گياهى نيز بيشترين درصد

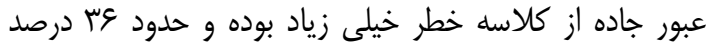

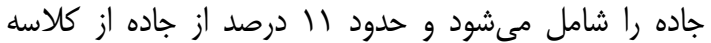

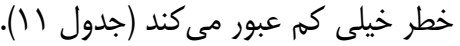

در بررسى جاده عبورى در نقشه كلاسابندى خطر

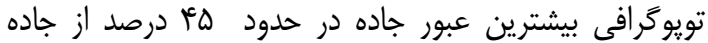

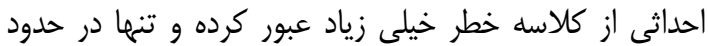

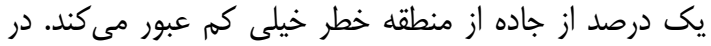

Table 11. Landslide hazard zonation results in soil criteria

جدول ||(- نتايج يهنهبندى خطر زمين لغزش در معيار خاى

\begin{tabular}{|c|c|c|c|}
\hline طول جاده (متر) & درصد عبور جاده & مساحت هكتار & كلاسه خطر \\
\hline $1 \cdot V T / 9 q+r \cdot r$ & $T / \Delta \cdot V T / Y A T \Delta$ & $r A F / I T$ & خطرخيلى كم \\
\hline $\mid V V^{e} \vee / q \vee \Lambda \mu \Delta D$ & D/VIETAIETV & $F \Delta F / A F$ & خطر كم \\
\hline & rr/\&MMQDTr & QRY/Ar & خطر متوسط \\
\hline $1 \cdot \Delta V V / \Delta q \cdot \Delta \Lambda$ & rE/DAFqTVGA & $9 q 4$ & خطر زياد \\
\hline SMN/Q.YAMI & Tr/D. KETTYY & $V T \Delta / g T^{C}$ & خطر خيلى زياد \\
\hline
\end{tabular}

كلاسههاى خطر متوسط، خطر زياد و خطر خيلى زياد عبور

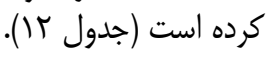

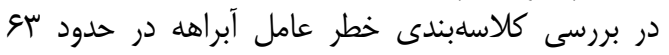

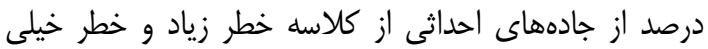

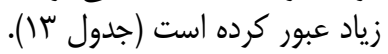

در بررسى جدول كلاسهبندى خطر از نظر زمين شناسى ادهى

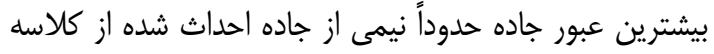

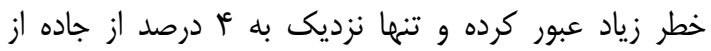

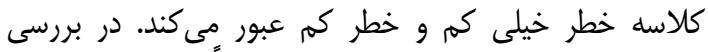

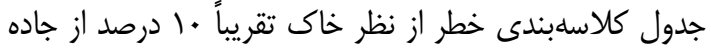

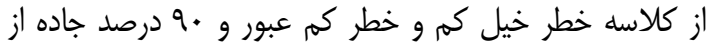

Table 12. Landslide hazard zonation results in geological criteria

جدول rا - نتايج يهنهبندى خطر زمين لغزش در معيار زمين شناسى

\begin{tabular}{|c|c|c|c|}
\hline طول جاده (متر) & درصد عبور جاده & مساحت هكتار & كلاسه خطر \\
\hline$\| T r / \cdot r \Delta \cdot r V$ & $r / V V r q 4 \cdot q .9$ & TFT/FY & خطرخيلى كم \\
\hline$V \varepsilon / F V V \Psi V \cdot \wedge r$ & $\cdot / T \Delta V \cdot \cdot r \Delta S G$ & $s q \cdot / \Lambda f$ & خطر كم \\
\hline$V \cdot \Delta \cdot /$ VQTKEY & $r$ r/gQR.NETr & gq4/Vr & خطر متوسط \\
\hline $\mid 1 \& 9 V / q 9111$ & rq/rTMMIqT & $\mid r \wedge 9 / T \Lambda$ & خطر زياد \\
\hline GNTN/MMQIV & $r$ r/qrg|gाII & GIV/rA & خطر خيلى زياد \\
\hline
\end{tabular}

Table 13. Results landslide hazard zonation in the criterion stream

جدول سا - نتايج يهنهبندى خطر زمين لغزش در معيار آبراهه

\begin{tabular}{|c|c|c|c|}
\hline طول جاده (متر) & درصد عبور جاده & مساحت هكتار & كلاسه خطر \\
\hline- & - & IIV/ צ & خطر خيلى كم \\
\hline GKGY/DVEqVq & $r \cdot|\Lambda| r \Delta V \Lambda \cdot \varphi$ & VEN/FG & خطر كم \\
\hline FV.r/q)MAVE & $\mid D /$ GTAFIDA| & GMN/T & خطر متوسط \\
\hline$q+\Lambda V / G V \Psi \mid G T$ & 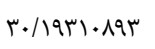 & IIFF/VT & خطر زياد \\
\hline Q & 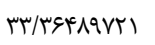 & $9 F N / 9 \Lambda$ & خطر خيلى زياد \\
\hline
\end{tabular}

لغرشهاى ثبت شده را شامل مى شود كه اين موضوع در اين

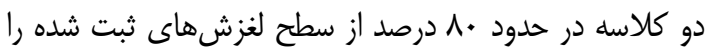

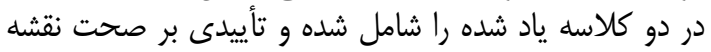

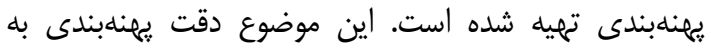

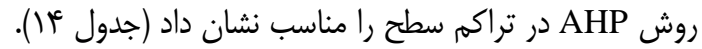

Table 14. Results zoning Landslide

در بررسى جدول كلاسهبندى خطر نهائى كه از نقشه

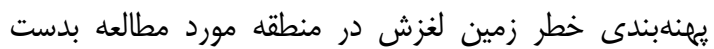

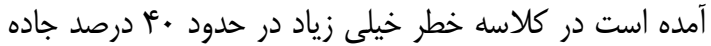

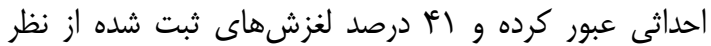

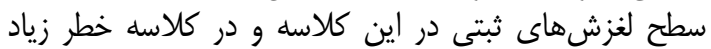

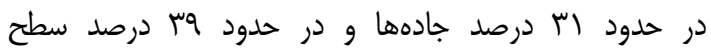
جدول fا - نتايج يهنهبندى نهائى خطر زمين لغزش

\begin{tabular}{|c|c|c|c|c|c|}
\hline طول جاده (متر) & درصد عبور جاده & تعداد بيكسل زمين لغزش & درصد وقوع زمين لغزش & مساحت هكتار & كلاسه خطر \\
\hline $9 / 91 \% \cdot \Lambda \Lambda V F \wedge$ & 1. & 1 & .1 .99 .91149 & $T F / \Delta G$ & خطرخيلى كم \\
\hline 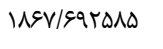 & S/TMMTRKE & ru & T/GNQVTIFTT & $9 / r / 8 \wedge$ & خطر كم \\
\hline$Q T+r / T \Delta \cdot \Delta T \&$ & 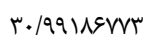 & fit & 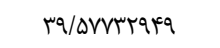 & $11 N / 9 T$ & خطر زياد \\
\hline IIqme/TVqTe & $F \cdot / \backslash A F \& \Delta F I q$ & erq & FI/TI. TVEeq & $r \Lambda T / \cdot r$ & خطر خيلى زياد \\
\hline
\end{tabular}




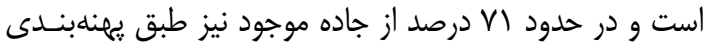

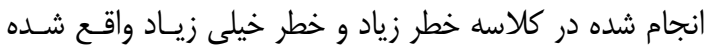

است (شكل r).

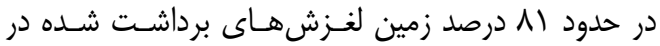

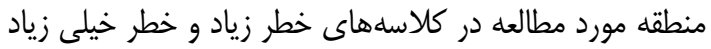

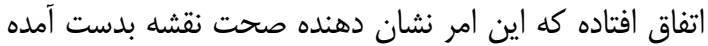

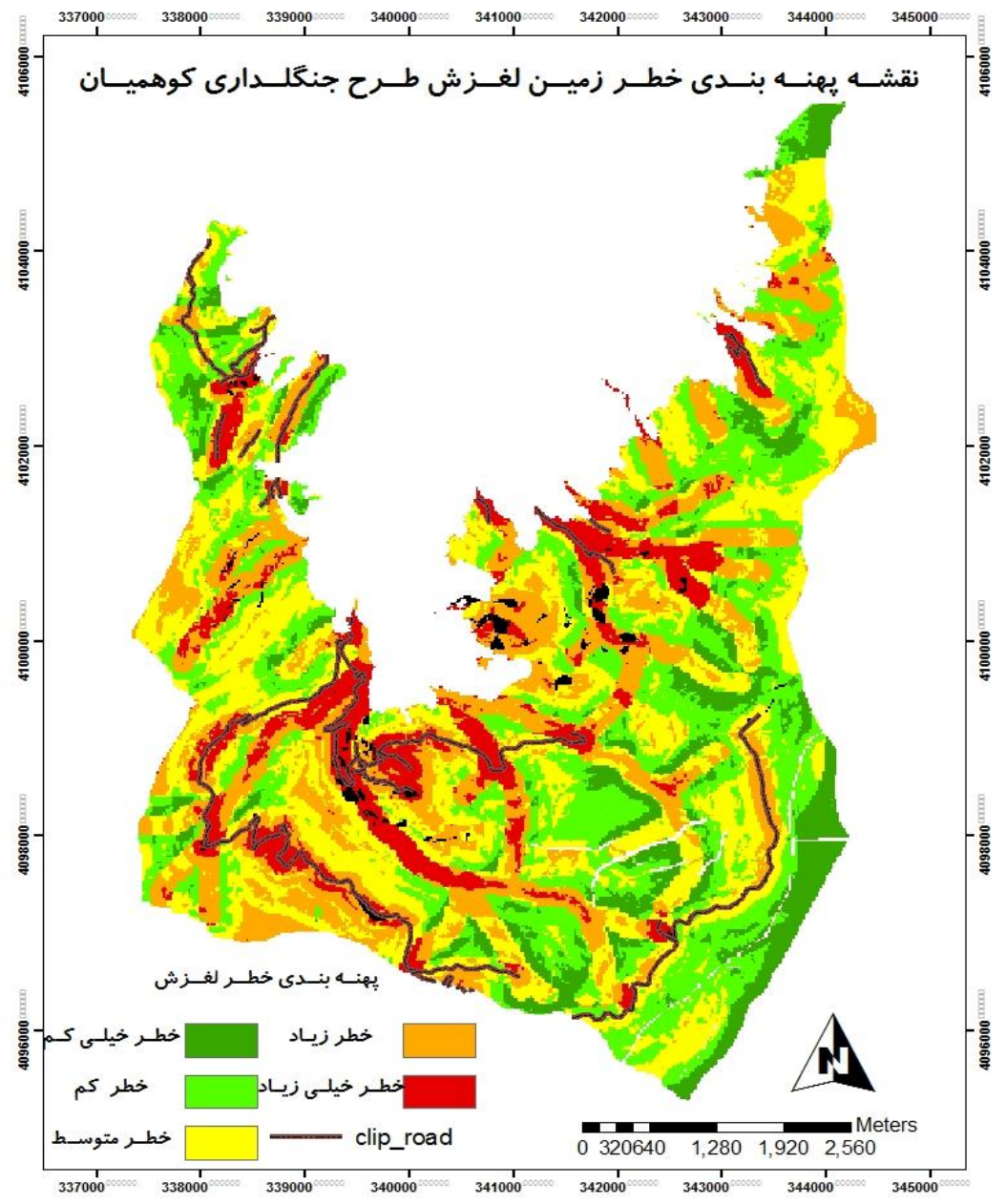

شكل r- نقشه يِنهابندى خطر زمين لغزش در طرح جنحَلدارى كوهميان

Figure 2. Landslide hazard zonation map in Koohmian forestry plan

ساير فعاليتهاى طرح را نيز تحت الشعاع قرار خواهد داد و

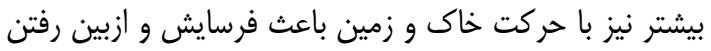

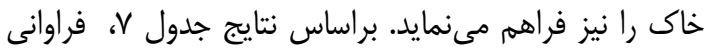

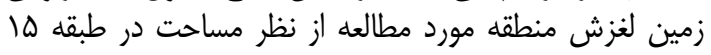

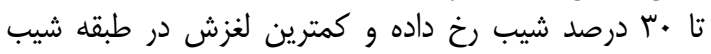

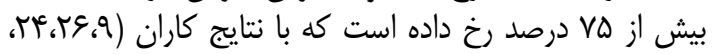

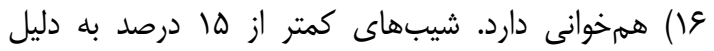

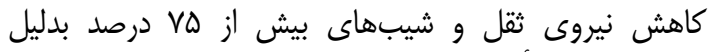

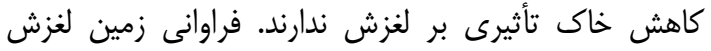

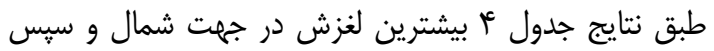

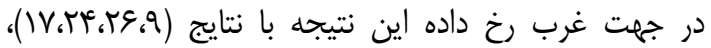

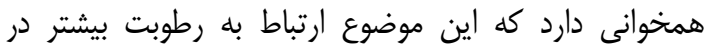

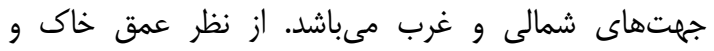

در اين يثوهش به بررسى عوامل مؤثر در خطر زمين

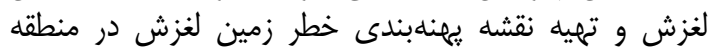

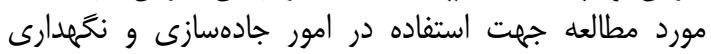

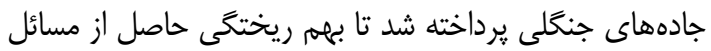

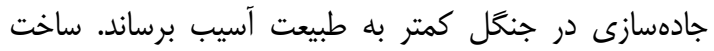

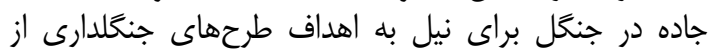

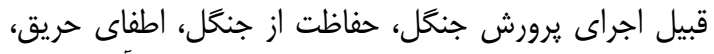

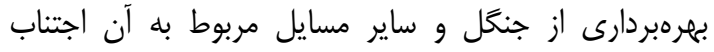

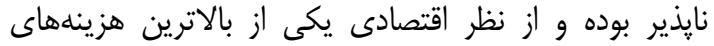

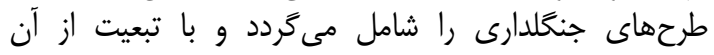

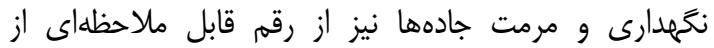

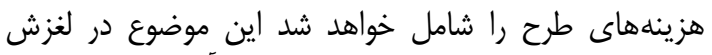

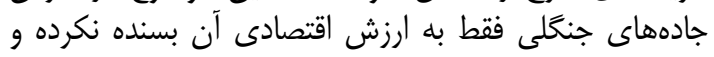




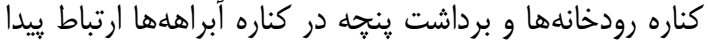

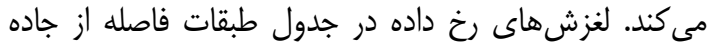

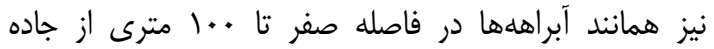

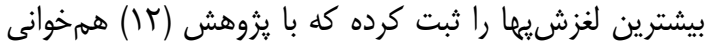

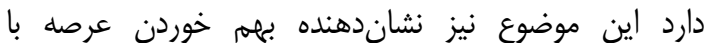

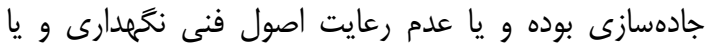

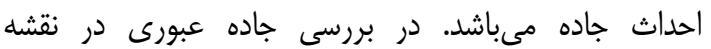

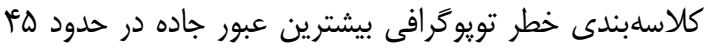

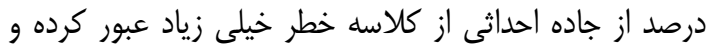

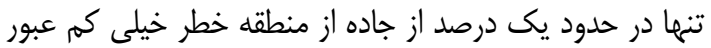

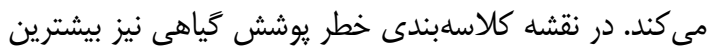

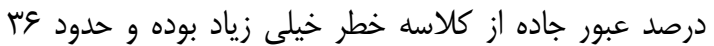

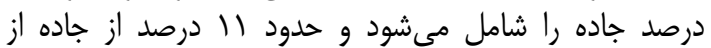

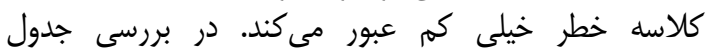

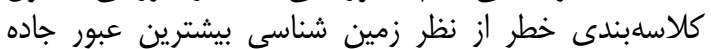

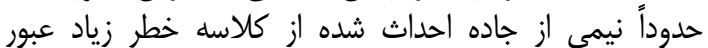

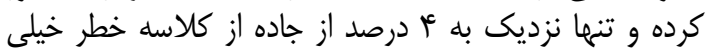

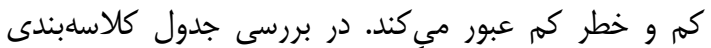

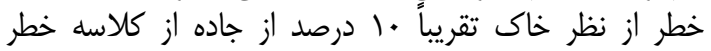

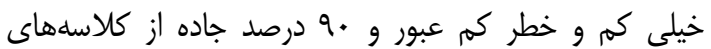

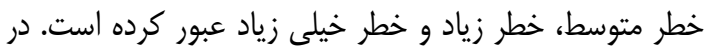

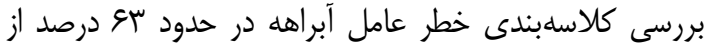

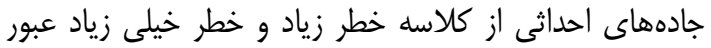

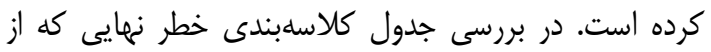

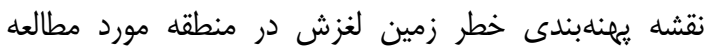

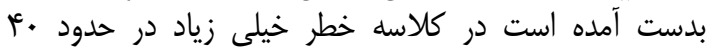

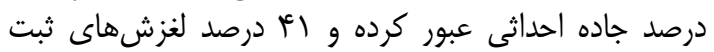

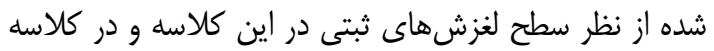

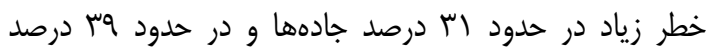

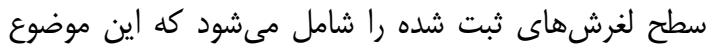

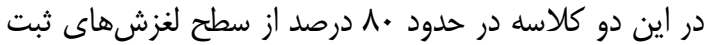

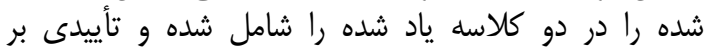

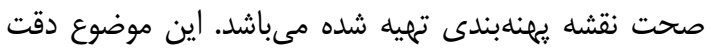

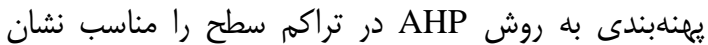

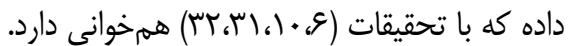

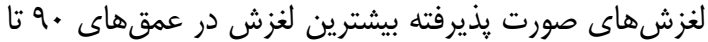

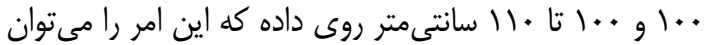

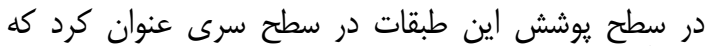

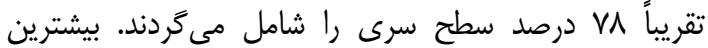

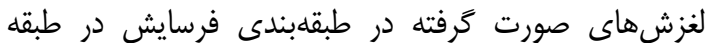

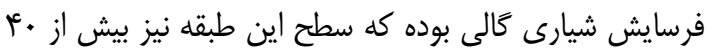

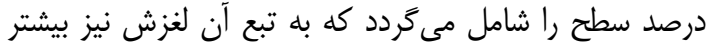

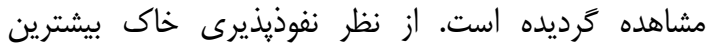

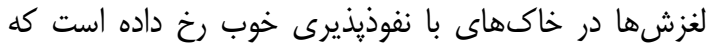

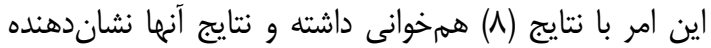

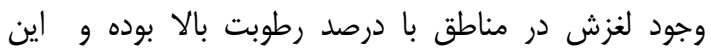

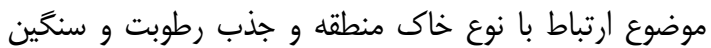

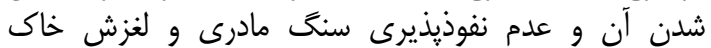

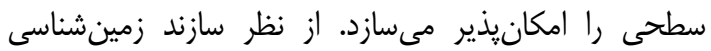

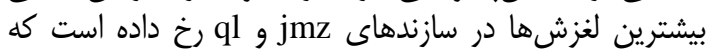

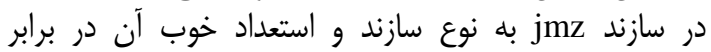

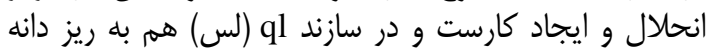

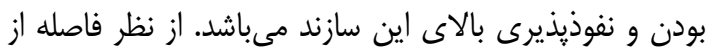

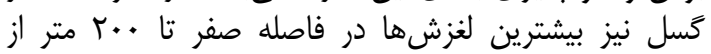

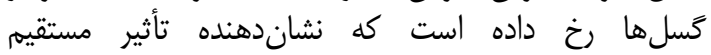

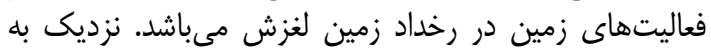

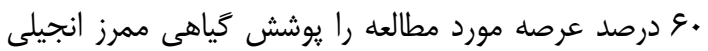

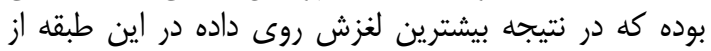

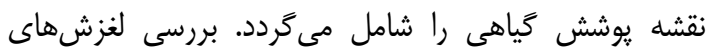

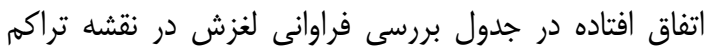

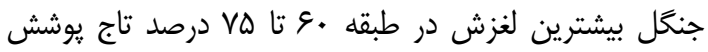

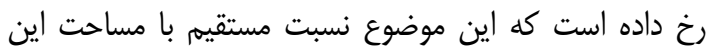

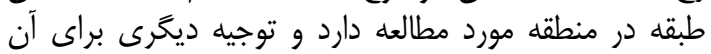

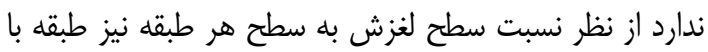

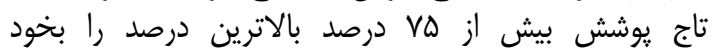

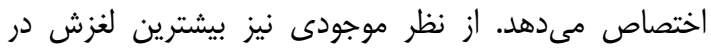

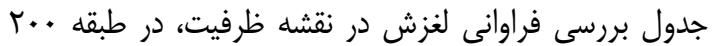

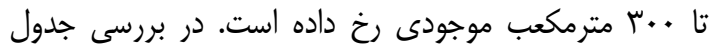

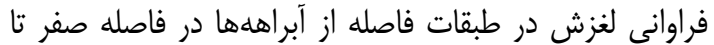

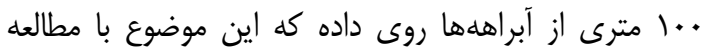

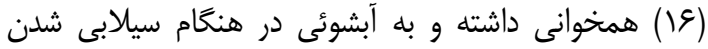

1. Afjeh Nasrabadi, H., Sh. Shatay, N. Raftnya and M. Sharia Ja'fari. 2008. Evaluation of the efficiency of statistical models of information value and surface density in landslide hazard zonation in forest areas (Series in Shasta Kalateh Forest of Gorgan). Journal of Agricultural Science and Natural Resources, 15(6) (In Persian).

2. Abdi, A. 2005. Forest road network design with minimum construction cost using GIS, Master's thesis, University of Tehran, $83 \mathrm{pp}$ (In Persian).

3. Akay, A. 2006. Minimizing Total Costs of Forest Roads with Computer-Aided Designmodel. Sadhana, 31: 621-633.

4. Akgun, A., C. Kıncal and B. Pradhan. 2012. Application of remote sensing data and GIS for landslide risk assessment as an environmental threat to Izmir city (west Turkey). Environmental Monitoring and Assessment 184, 9: 5453-5470.

5. Aksoy, B. and M. Ercanoglu. 2012. Landslide identification and classification by object-based image analysis and fuzzy logic: An example from the Azdavay region (Kastamonu, Turkey). Computers \& Geosciences, (38)1: 87-98.

6. Abdul Khani, A. and A.S. Jamali. 2012. Application of GIS and Analytical Hierarchy Process in Landslide Risk Mitigation and Comparison of the Preference of Factors Influencing Slippage - Case Study: Mangashed Watershed Yazd. Conference and Exhibition Geotechnical, 88 (In Persian). 
7. Arabameri, A., K. Shirani and K. Rezaeei. 2017. A Comparative Assessment between Weights-ofEvidence and Frequency Ratio Models for Landslide Hazard Zonation in Vanak Basin. Journal of Watershed Management Research, 15: 147-160 (In Persian).

8. Abedi, T., S.A. Hoseini and R. Naghdi. 2010. Study of Relationship between Soil Mechanical Characteristic and Landslide in Forest Road Route (Case Study: Chafroud Watershed Guilan Province), Watershed Management Research, 1(1): 17-29 (In Persian).

9. Ahmadi, E., A. Dervish adjective, M. Makhdoom and Sh. Abolqasemi. 2005. Road Routing Based on Environmental GIS Principles Using Geomatics Conference 84, 8 pp (In Persian).

10. Anonymous. 2011. Kohimish forestry revision plan.

11. Buia, D.T., B. Pradhanc, O. Lofmana, I. Revhauga and O.B. Dicka. 2012. Landslide susceptibility mapping at Hoa Binh province (Vietnam) using an adaptive neuro-fuzzy inference system and GIS. Computers \& Geosciences, 45: 199-211.

12. Kalarstagi, A.S., M. Habib Nejad and H. Ahmadi. 2007. Study of landslides occurring in relation to land use change and road construction Case study of Tajan watershed, Sari. Geographic Research, 62 (86): 91-81 (In Persian).

13. Fattahi Bandpei, M., M. Ghafouri, Gh. Lashkaripoor and N. Hafzii Moghads. 2012. Locating the Pathway Band-Banay Road in the south of Neka using GIS. Practical Geology Quarterly, 2: 123-111 (In Persian).

14. Graee, P., H. Karimi and M. Timurid. 2011. Landslide hazard zonation in Vavanest watershed - Sari. Seventh National Conference on Watershed Engineering Sciences, 8 pp (In Persian).

15. Graee, P., H. Karimi and M. Tavakoli. 2007. Landslide hazard zonation and determining the factors affecting it in the Ilam Dam area. Water and Soil Science, 53: 99-111 (In Persian).

16. Hayati, A., B. Majnonian, A. Abdi, A. Dastrang and A.A. Nazari Samani. 2012. Landslide risk zoning for use in the design of forest road network. Journal of Forest and Wood Products, Iranian Journal of Natural Resources, 65(1): 19-32 (In Persian).

17. Hosseini, S.A. 1993. Study of the landslide phenomenon on mountainous roads, Master's Thesis, Faculty of Natural Resources, Tarbiat Modares University. 98 p. Faculty of Natural Resources, Sari, $60 \mathrm{pp}$ (In Persian).

18. Hosseini, S.A., R. Lotfi, M. Lotfalian, A. Kavian and A. Parsakhoo. 2011. The effect of terrain factors on landslide features along forest road African Journal of Biotechnology, 10(64): 1410814115 .

19. Kamani, M. and M. Dadkhah. 2011. Investigating the role of the slope in the zoning of the risk of landslide and eliminating the errors of the collected articles of the thirteenth congress of earth sciences from 1 to 3 March 2011.7 pp (In Persian).

20. Kanungo, D.P. and S. Sarkar. 2003. Landslides and terrain parameters in Darjeeling Himalaya. Jour. of Himalayan Geology, Dehradun, (24): 55-62.

21. Motavaly, M., R. Ismaili and M.M. Hosseinzadeh. 2009. Determination of landslide landslide sensitivity using logistic regression in Mazandaran province basin. Natural History Quarterly, 2(5): 73-83 (In Persian).

22. Mousavi Khatir, S.Z., A. Kavian and K. Solimani. 2010. Preparation of landslide susceptibility map in Sajavand watershed using logistic regression model. Journal of Agricultural Science and Technology of Natural Resources, Water and Soil Science, 53: 99-111 (In Persian).

23. Mohammadi, M., H. Pourghasemi and P. Feyznaya. 2010. Analyzing and estimating landslide hazard using the hierarchical analysis process method in a part of the road of Haraz. Moderator of Human Sciences - Space Planning and Planning, 14(2): 155 pp (In Persian).

24. Mohammadi, M. and A. Pourghasemi. 2017. Prioritization of Landslide-Conditioning Factors and its Landslide Susceptibility Mapping using Random Forest New Algorithm, Journal of Watershed Management Research, 8(15): 161-170 (In Persian).

25. Pourghasemi, H., G. Jirandeh, A. Pradhan, B. Chong xu and C. Gokceoglu. 2013. Landslide susceptibility mapping using support vector machine and GIS at the Golestan Province, Iran. Journal of Earth System Science, 122(2): 349-369.

26. Pradhan, B., A. Chaudhari, J. Adinarayana and M.F. Buchroithner. 2012. Soil erosion assessment and its correlation with landslide events using remote sensing data and GIS: a case study at Penang Island, Malaysia. Environmental Monitoring and Assessment, 184(2): 715-727.

27. Rafta Nia, N., M. Kavianpour and T. Ahmadi. 2011. Investigating the causes of landslide phenomenon in Golindrood Forest (Case Study, Series 3 of Watershed Area 48). Journal of Science and Technology of Natural Resources, 6(1): 63-53 (In Persian).

28. Rafta Nia, N., M. Kavianpour and T. Ahmadi. 2010. Study of the causes of landslide in Glendrood forest (Case study, Series 3, Watershed Area 48), Journal of Natural Resources Science and Technology, 6(1): 53-63.

29. Sarikhani, N. and B. Majnanian. 2005. Guidelines for the design, implementation and exploitation of forest roads. Journal Publications of Planning and Budget Organization, 131:170 pp (In Persian).

30. Shadfar, P. and M. Yamani. 2007. Landslide hazard zonation in the Jalisen watershed using the LNRF model. Geographic Research, 62: 11-23 (In Persian).

31. Yalcin, A. 2008. GIS-based landslide susceptibility mapping using analytical hierarchy process and bivariate statistics in Ardesen (Turkey): Comparisons of results and confirmations, Catena, 1-12.

32. Zare, M., H. Ahmadi and Sh. Gholami. 2010. Landslide hazard assessment using multi-criteria decision making process and Geographic Information System (GIS) Case Study, Waz Basin. Scientific Papers of Natural Ecosystems of Iran, 1(2): 179-168 (In Persian). 


\title{
Investigation of Landslide Ranger Zoning using Analytical Hierarchy Process in GIS Environment (Case Study: Azadshahr Kohmian Forestry Design)
}

\author{
Abdolmajid Jarjani ${ }^{1}$, Hasan Akbari ${ }^{2}$, Seyyed Ata Hosseini ${ }^{3}$ and Omid Abdi ${ }^{4}$ \\ 1- Graduted M.Sc. Student, In Forest Engineering, Faculty of Natural Resources, Sari Agricultural Sciences and \\ Natural Resources University \\ 2- Assistant Professor, Faculty of Natural Resources, Sari Agricultural Sciences and Natural Resources University \\ (Corresponding author: hassan_akbarivas@yahoo.com) \\ 3- Professor of Forestry and Forest Economics Department of Tehran University, Karaj \\ 4- Graduted M.Sc. Student, Forest Sciences Faculty, Gorgan Agricultural Sciences and Natural Resources University \\ Received: December 5, 2017 \\ Accepted: August 7, 2018
}

\begin{abstract}
Landslide is one of the destructive phenomena of natural resources and threatens the sustainability of forest roads. The purpose of this study is to determine the zoning of land risk for the stability of forest roads using the process of analyzing the hierarchy in the GIS. The final zoning map of landslide hazard based on the combination of different maps and main criteria of topography, vegetation, geology, soil, waterways and roads, in 5 classes of landslide danger $(40.19 \%)$, high risk $(30.99 \%)$, medium risk $(22.5 \%)$, low risk $(6.29 \%)$ and very low risk $(0.03 \%)$. According to the results, the maximum area of landslide in terms of slope (15-30\%), in terms of direction (north direction), in terms of soil depth (100-110 cm depth), soil permeability (with good permeability), in terms of The geology ( $\mathrm{jmz}$ and ql formations), in terms of distance from the fault $(0-200 \mathrm{~m})$, is recorded in terms of distance from the road $(0-100 \mathrm{~m})$ in terms of distance from the road $(0-100 \mathrm{~m})$. Which is based on the AHP process of accuracy.
\end{abstract}

Keywords: Analytical Hierarchy process (AHP), Geography information system (GIS), Main criterion, Road forest, Slippage, Zoning map 